\title{
TECHNICAL REVIEW ON MACHINE LEARNING
}

\author{
Shrushti Manish Ambulgekar \\ Computer Science and Engineering Department \\ P.E.S College of Engineering, Aurangabad
}

\begin{abstract}
Machine learning is sub-branch of Artificial intelligence and Deep learning is sub-branch of Machine learning. In the machine learning computer has input as past experience and some task from which performance of machine increases. There are 3 types of machine learning i.e. 1) Supervised machine learning 2) Unsupervised machine learning and 3)
\end{abstract}

Reinforcement machine learning. Each type has its own problems and Applications. Machine learning has its own Applications in technical as well as in non-technical fields. There is a vast future of Machine learning in upcoming years like use in Army, Hospitals. The machine learning field, which can be briefly defined as enabling computers make successful predictions of past experiences, has shown an impressive development recently with the help of the faster increase in the storage capacity and processing power of computers [1]. Together with many other disciplines, machine learning methods have been widely employed in bioinformatics [1].

Keywords: - Machine Learning, Supervised machine learning, Unsupervised machine learning and Reinforcement machine learning.

\section{INTRODUCTION}

Machine learning (ML) is the study of computer algorithms that improve automatically through experience [2]. It is seen as a subset of artificial intelligence [2]. Machine learning algorithm is build a mathematical model which based on sample data, known as "training data"[2], in order to make predictions or decisions without being explicitly programmed to do so. Machine learning algorithms are used in a wide varieties of applications, such as email filtering and product recommedation, and it is difficult as well as infeasible to develop simple and understandable algorithms to perform the needed tasks.

History:- In 1837 Charles Babbage invented Analytical Engine, Lady Ada Lovelace was friend of Charles Babbage, Lady Ada Lovelace made statement about computer or Analytical Engine in 1843 that "The Analytical Engine has

\author{
Prajakta Dashrath Mali \\ Computer Science and Engineering Department \\ P.E.S College of Engineering, Aurangabad
}

no pretension whatever to originate anything, It can do whatever we Know how to order it to perform. Its providence is to assist us in making available what we've already acquainted with." (Computers can't work on its own) Alan Tuning did research on this Analytical engine and after that research he took objection on statement of Lady Ada Lovelace and stated that Computer can work on its own.

Machine Learning:- Machine Learning is subbranch Artificial Intelligence while Deep Learning is sub-branch of Machine Learning. Machine Learning and Deep Learning together make Artificial Intelligence [3]. Machine Learning is a set of past experience algorithm and which improve from this experienced data [4]. Machine Learning algorithm works on training data and is used for Predictions or decisions [5]. Machine Learning algorithm is used wide range of applications like computer vision and email filtering. Machine Learning is related to computational statistics, focuses on predictions. Tom Mitchell formal definition "A computer program is said to learn from experience $\mathrm{E}$ with respect to some class of task $\mathrm{T}$ and performance measure $\mathrm{P}$ if it's performance at task in $\mathrm{T}$ as measured by $\mathrm{O}$, improves with experience E"[6]. Machine Learning has 3 forms [7] 1) Supervised machine learning 2) Unsupervised machine learning and 3) Reinforcement machine learning.

1. Supervised Machine Learning: In this approach the system is trained on predefined set of training, examples which then facilitate its ability to reach an accurate conclusion when given new data. Supervised Learning can be mathematically be defined as $\mathrm{Y}=\mathrm{f}(\mathrm{x})$. The goal is to approximate the prediction. Problems of SML are Classification and Regression. Classification problem is when output variable is a category such as 'red' or 'blue' or 'disease' or 'non-disease', it is discrete value. While Regression problem is when the output variable is real value, Such as 'dollar' or 'weight' mathematically given example: of function $\mathrm{f}(\mathrm{Xf}(\mathrm{X}))$ prediction function $\mathrm{f}(\mathrm{X})$, Regression is continuous value. Applications of SML are 1)

Prediction analysis based on regression or categorical classification. 2) Spam detection. 3) Pattern detection. 4) Natural language processing. 5) 


\section{International Journal of Engineering Applied Sciences and Technology, 2020 \\ Vol. 5, Issue 6, ISSN No. 2455-2143, Pages 183-187 \\ Published Online October 2020 in IJEAST (http://www.ijeast.com)}

Sentiment analysis. 6) Automatic image classification. 7) Automatic sequence processing. (eg : speech or music). Examples of SMLA are 1) Linear regression. 2) Random forest for classification and regression. 3) Support vector machine for classification.

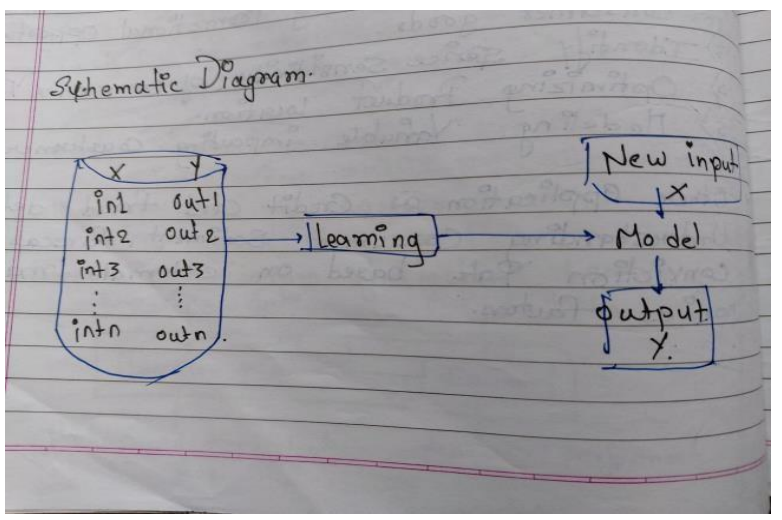

Fig 1. Supervised Machine Learning.

\section{Unsupervised Machine Learning:}

Unsupervised Machine Learning means absence of supervisor. It is useful when it necessary to learn how a set of element can be grouped. According to their similarity. We have input (x) and no corresponding output variables. The goal for USML is the model the underlying structure or distribution in the data in order to learn more about the data. Problems of USML are Clustering and Association. Clustering is the problem where inherent grouping in the data are discovered such as grouping customers by purchasing behavior. Applications are 1) Similarity detection 2) Automatic labeling 3) Object segmentation.

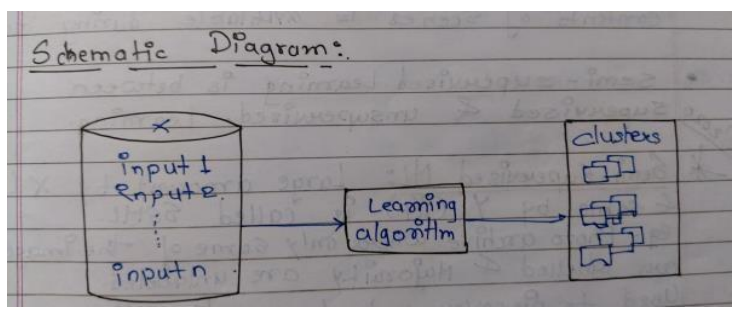

Fig 2. Unsupervised Machine Learning

3. Reinforcement Learning: Kearns from feedback provided by the environment. Feedback is from of penalty or reward. Used to understand the action is positive or not. Decision in immediate and cumulative reward for high. Efficiently in not completely deterministic environment.
When very dynamic and when it's impossible to have a precise error measure. Applications are 1) Traffic light control. 2) Robotics. 3) Personalized Recommendation.

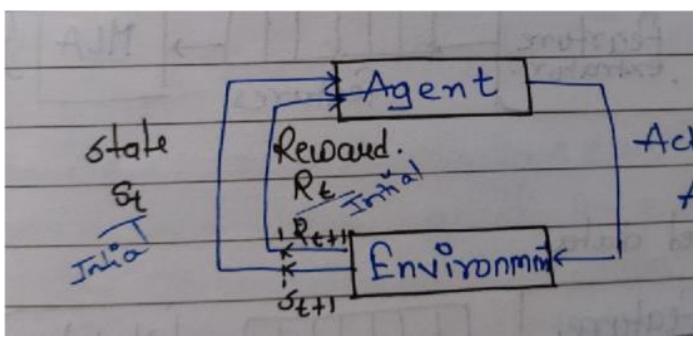

Fig 3. Reinforcement Machine Learning.

Applications: 1) Medicine 2) Computer vision 3) NLPNatural language processing 4) Business intelligence.

Research related to Machine Learning is important because, Machine learning has big future and importance in human life. Machine Learning is very vast topic and this field is developing day by day, new research, new inventions and new innovations. We can also say this field is also developing with time, every day, every month something new invention is coming to light. Doing research in this field means digging into the future of humans and future of country. There is big and vast future scope of Machine learning. In future there may will be small home working Robots with the help of Artificial intelligence which will be working at home doing home related works, also these robots can be seen working in Malls, Hospitals, Hotels, Research centers, etc. The latest example of robotics are robot created by ISRO Indian space research center named Vyommitra (Female Shaped Robot), Which is going to help our scientist in study, Research on planets. One more example during this Global Pandemic also some Robots are severing patients in hospitals for medicines, food and for checking their health updates. In robotics machine learning plays most import role in making them learn and improve their experience

This field is not only related to robotics but also Speech recognition as, The Latest example of this Speech recognition is Alexa of Amazon, Google Assistant of Google, Sire of Apple iPhone, and Bixby of Samsung. This devices reply on our speech. Here input and output both are Speech, they recognition our speech then decode and find the information which is asked and the give output in Speech. This Speech recognition is also used in crime investigation, like if officers found out some audio recording then officers can give input to device audio of suspects and that device will try to match that audio with found out audio and will give output. Not only in this field but also in medical field as well, there data is given as symptoms of disease and input of 


\section{International Journal of Engineering Applied Sciences and Technology, 2020 \\ Vol. 5, Issue 6, ISSN No. 2455-2143, Pages 183-187 \\ Published Online October 2020 in IJEAST (http://www.ijeast.com)}

voice of patient and device match that symptoms with voice and tells whether patient is infected or not.

In space Satellite can be define as SML robots which work on different surfaces and the places where humans can't work. Here the input is give as instructions to them to collect those samples and as output send them to research center for study purpose. In future this Machine Learning is going to be big treat for human race, they will also be used in Army, like for detecting the tourist activities, finding bombs and etc.

\section{APPLICATION USING MACHINE LEARNING}

1. Product recommendation: Machine learning is used by various e-commerce and entertainment companies like Amazon, Netflix, Hot star, etc. for product recommendation to users. Whenever user search for some product we get different Advertisement for same product with different price and sellers. Google understand the interest of the user, by using different machine learning algorithm and recommend the product as per user's interest. Same happens with Netflix, Amazon prime suggest about different series, movies as per our search and interest.

2. Self-Driving Car: One of the most interesting feature or application of Machine Learning is Self-driving Cars. Machine Learning plays important role in self-driving cars. The well Car manufacturing company that is Telsa, is working on self-driving car.

It is using UN supervising Machine Learning method to train their car to detect obstacles to avoid it and work smoothly.

3. Virtual Personal Assistants: Sire, Alexa, Google Now are some of the popular examples of virtual personal assistants. As the name suggests, they assist in finding information, when asked over voice. All you need to do is activate them and ask "What is my schedule for today?", "What are the flights from Germany to London", or similar questions. For answering, your personal assistant looks out for the information, recalls your related queries, or send a command to other resources (like phone apps) to collect info. You can even instruct assistants for certain tasks like "Set an alarm for 6 AM next morning", "Remind me to visit Visa Office day after tomorrow. Virtual Assistants are integrated to a variety of platforms. For example, Smart Speakers:

Amazon Echo and Google Home, Smartphones: Samsung Bixby on Samsung S8, Mobile Apps: Google Allo.

\section{Predictions while Commuting: Traffic} Predictions: We all have been using GPS navigation services. While we do that, our current locations and velocities are being saved at a central server for managing traffic. This data is then used to build a map of current traffic. While this helps in preventing the traffic and does congestion analysis, the underlying problem is that there are less number of cars that are equipped with GPS. Machine learning in such scenarios helps to estimate the regions where congestion can be found on the basis of daily experiences. Online Transportation Networks: When booking a cab, the app estimates the price of the ride. When sharing these services, how do they minimize the detours? The answer is machine learning. Jeff Schneider, the engineering lead at Uber ATC reveals in an interview that they use ML to define price surge hours by predicting the rider demand. In the entire cycle of the services, ML is playing a major role.

2. Email Spam and Malware Filtering: There are a number of spam filtering approaches that email clients use. To ascertain that these spam filters are continuously updated, they are powered by machine learning. When rulebased spam filtering is done, it fails to track the latest tricks adopted by spammers. Multi-Layer Perceptron, C 4.5 Decision Tree Induction are some of the spam filtering techniques that are powered by ML. Over 325, 000 malwares are detected every day and each piece of code is 90-98\% similar to its previous versions. The system security programs that are powered by machine learning understand the coding pattern. Therefore, they detects new malware with $2-10 \%$ variation easily and offer protection against them.

3. Online Fraud Detection: Machine learning is proving its potential to make cyberspace a secure place and tracking monetary frauds online is one of its examples. For example: PayPal is using ML for protection against money laundering. The company uses a set of tools that helps them to compare millions of transactions taking place and distinguish between legitimate or illegitimate transactions taking place between the buyers and sellers.

\section{LITERATURE REVIEW}

Hua et at an Author, In 2006 [8] Propose the support vector machine approach for predict of occurrence for nonzero demand or load time demand of spare parts which used in petrochemical enterprise in china for inventory management. It can used in integrated procedure for establishing a correctional of explanatory variables and autocorrelation of time series demand which demand of spare parts. After performing the comparison the performance of SVM model with LRSVM model, Creston's model, exponential smoothing model, IFM method and Markov bootstrapping procedure, it's result came out as SVM model performs best than others.

Wang, in 2007 [9] described Machine Learning with Genetic Algorithm, with real Gas. In this experiment releases came out that, SVR shows the ARIMA model and 


\section{International Journal of Engineering Applied Sciences and Technology, 2020 \\ Vol. 5, Issue 6, ISSN No. 2455-2143, Pages 183-187 \\ Published Online October 2020 in IJEAST (http://www.ijeast.com)}

BPNN regards the based on normalized mean square error and Absolute mean percentage error.

Foully ET at an author, in 2018 [10] suggested that statistical method employing Machine learning model and to analyze and can apply it to solar irradiation prediction working hourly. This methodology uses the high, low and medium meteorological variability like Ajaccio, Odell and Tiles. Also compared the model with auto regressive moving and average and multi-layered preceptor.

Makridakis , In 2018 [11] present Machine Learning as methods as Statistical time series forecasting and compare the correctness of that methods with correctness of conventional statistical method, found that first one is better and output using both the measure accuracy . They provide reason for accuracy of learning model is less than statistical model and suggest some more methods and ways.

\section{TOOLS USED FOR MACHINE LEARNING.}

Tools which are used in machine learning makes machine learning rapid and swift. The interface for machine learning programming language is given by tools. The process and implementation is provided by Machine learning [12]. The platform which provides capabilities to run model and project. Some examples are:

$$
\begin{array}{ll}
- & \text { R platform. } \\
\text { - } & \text { Python Spicy. } \\
\text { WEKA machine learning platform. }
\end{array}
$$

These tools also contain various laborites for running the model and project. Some examples are:

$$
\begin{array}{ll}
- & \text { scikit of python } \\
- & \text { JAST of JAVA }
\end{array}
$$

\section{CONCLUSION}

In this paper there is short history of Machine learning with origin of it from Charles Babbage inventing Analytical Engine, Lady Ada Lovelace statement to Alan tuning object and purposing that computer can think on its own. Also in Introduction part of this paper there are different types of Machine learning with their applications, problems and definition. This part also contains the future scope of Machine learning in different as well as use of it in different fields [13]. In literature review we has seen different research done on different topics using different machine learning methods. Even we have seen different applications which are useful for this century and some tools of machine learning.
Machine learning approaches applied in systematic reviews of complex research fields such as quality improvement may assist in the title and abstract inclusion screening process. Machine learning approaches are of particular interest considering steadily increasing search outputs and accessibility of the existing evidence is a particular challenge of the research field quality improvement. Increased reviewer agreement appeared to be associated with improved predictive performance[14][15][16].

\section{REFERENCES}

1. https://legaltechcenter.openum.ca/files/si tes/159/2020/05/Ethical-Issues-in-AIpowered-LegalTech.pdf

2. https://link.springer.com/protocol/10.100 7/978-162703-748-8_7

3. Brownlee Jason in (November 25, 2013) "A Tour of Machine Learning algorithms"

4. www.cs.cmu.edu. (Retrieved 2020-05-28) "Machine Learning Textbook".

5. www.deeplearning.org "Deep Learning", .

6. Author Samuel (1996): The definition "Without the explicitly programmed" is often attributed with, who coined the term Machine Learning in 1959, this phrase is not found verbatim in the publication and may be paraphrase that appeared later. The Conference of "paraphrasing of Author Samuel in 1959 and the question is : How 14.

https://en.wikipedia.org/wiki/Machine_le can computer learn to solve problem arning without explicitly programmed? " in Koza, John R; Benneth, Forrest H; Ander, David

;Keane, Martin A in 1996. Automated Design of Both the Topology and Sizing of Analog Electrical Circuits Using Genetic Programming. Artificial Intelligence in Spinger, Dordrecht.pp.151- 170.doi:10.1007/978-94009-0279-4_9.

7. Bishop. C. M (2006) Pattern Recognition and Machine learning, Springer, .ISBN 978-0-387-31073--2.

8. "The facets of same field can be" Machine Learning and Pattern Recognition. ।

9. Real Carbonneau, Kevin Laframboise, Rustam Vahidov, (2008) "Application of Machine Learning techniques for supply chain demand forecasting" in European Journal of Operational Research 184, pp. 11401154.

10. Wei-Chiang Hong, Yucheng Dong, Li-Yueh Chen, Shih-Yung Wei (2011) " SVR with hybrid chaotic genetic algorithms for tourism demand forecasting" ,Applied Soft Computing 11, pp. 1881-1890.

11. Spyros Makridakis, Evangelos Spiliotis, Vassilios Assimakopoulos (2018) "Statistical and 
Machine Learning forecasting methods: Concerns and ways forward", PLoS ONE 13.

12. Yu Zhang, Yu Wang, Guoxu Zhou, Jing Jin, Bei Wang, Xingyu Wang, Andrzej Cichocki (2018) "Multi-kernel extreme learning machine for EEG classification in brain-computer interfaces" ,Expert Systems With Applications 96, pp. 302-310,

13. https://towardsdatascience.com/what-is-machine learning-and-types-of-machine- learning-andrewsmachine-learning-part-1 9cd9755bc647 